\title{
WMAP CONSTRAINTS ON A QUINTESSENCE MODEL *
}

\author{
T. BARREIRO, M. C. BENTO, N. M .C. SANTOS AND A. A. SEN \\ Departamento de Fúsica, Instituto Superior Técnico, Av. Rovisco Pais 1, 1049-001 \\ Lisboa, Portugal \\ E-mail:tiago@glencoe.ist.utl.pt,bento@sirius.ist.utl.pt,ncsantos@cfif.ist.utl.pt, \\ anjan@x9.ist.utl.pt
}

\begin{abstract}
We use the results from the Wilkinson Microwave Anisotropy Probe (WMAP) for the locations of peaks and troughs of the Cosmic Microwave Background (CMB) power spectrum, together with constraints from large-scale structure, to study a quintessence model in which the pure exponential potential is modified by a polynomial factor. Our analysis, in the $\left(\Omega_{m}, h, n_{s}\right)$ cosmological parameters space shows that this quintessence model is favoured compared to $\Lambda$ CDM for $n_{s} \approx 1$ and relatively high values of early quintessence; for $n_{s}<1$, quintessence and $\Lambda \mathrm{CDM}$ give similar results, except for high values of early quintessence, in which case $\Lambda \mathrm{CDM}$ is favoured.
\end{abstract}

Recent cosmological observations suggest that the dynamics of the Universe at the present is dominated by a negative pressure component, called dark energy. Various possibilities for the nature of this dark energy have been considered, notably the cosmological constant and quintessence, a dynamical scalar field leading to a time-varying equation of state parameter, $w_{\phi} \equiv p_{\phi} / \rho_{\phi}$. These models most often involve a single field 1 or, in some cases, two coupled scalar fields 2 . Other possibilities for the origin of dark energy include the generalized Chaplygin gas proposal 3 and Cardassian models 4 . In order to unravel the nature of dark energy, it is crucial to use observations so as to be able to discriminate among different models. In particular, the existence of a dark energy component affects the structure of the CMB power spectrum, which is particularly sensitive to the amount of dark energy at different epochs in cosmology. For instance, the locations of peaks and troughs depend crucially on the amount of dark energy today and at last scattering as well as the dark energy time-averaged equation of state, which are model-dependent quantities 5 . Hence, one can use the high-precision measurements recently obtained by the BOOMERanG $\frac{6}{6}$, MAXIMA-1 $\frac{7}{2}$, Archeops $\frac{8}{6}$ and, in particular, WMAP 9 observations to constrain dark energy models.

We study the effect of a dark energy component defined by the quintessence potential ${ }^{10} V(\phi)=\left[A+\left(\phi-\phi_{0}\right)^{2}\right] e^{-\lambda \phi}$ on the location of the first three peaks and the first trough of the CMB power spectrum. We have also analyzed the consequences of cluster abundance constraints 11 on $\sigma_{8}$, the $r m s$ density fluctuations averaged over $8 h^{-1} \mathrm{Mpc}$ spheres. This M-theory motivated potential leads to a model with some interesting features, namely there are two types of attractor solutions giving rise to an accelerating universe today, corresponding to permanent or transient acceleration 12. Transient vacuum acceleration is a particularly appealing scenario that would also solve the apparent incompatibility between an eternally

*TALK PRESENTED BY N. M. C. SANTOS AT THE TENTH MARCEL GROSSMANN MEETING ON GENERAL RELATIVITY

rioproc2: submitted to World Scientific on December 16, 2018 
accelerating universe and string theory, at least in its present formulation, given that string asymptotic states are inconsistent with spacetimes that exhibit event horizons 13 .

We should emphasize that restricting the analysis of the CMB power spectrum to the positions of peaks and troughs, rather than considering the structure of the whole spectrum, turns out to be a simple but very powerful tool in constraining the model parameters due to the high accuracy with which these positions are now determined, particularly after WMAP results. One should also notice that, although our study is limited to the $\left(\Omega_{m}, h, n_{s}\right)$ parameter space, we expect (and have, to some extent, checked) that these are the most influential parameters; of course, $\Omega_{b} h^{2}$ can also be important although not within the rather strict WMAP error bars, $\Omega_{b} h^{2}=0.0224 \pm 0.0009$.

In our study 14 , we used the accurate analytic approximations given by Doran and Lilley ${ }^{15}$ for the positions of the first three peaks and first trough. Notice that although those formulae were obtained using a standard exponential potential, one expects the results to be fairly independent of the form of the potential unless it is qualitatively very different from the exponential potential before last scattering.

We found that the dependence of the peaks locations on parameter $A$ is extremely small and can be safely neglected. As should be expected, changes in $A$ in order to get the transient or permanent acceleration regimes do not alter the analysis since the two regimes do not differ significantly until the present, and therefore peak positions should not be affected. For each value of $\lambda$ and $A$, $\phi_{0}$ is chosen such that $\Omega_{t o t}=1$. Hence, the model's behaviour depends essentially on parameter $\lambda$, which measures the amount of "early quintessence" 16 , the average fraction of dark energy before last scattering $\left(\bar{\Omega}_{\phi}^{l s} \sim 3 / \lambda^{2}\right)$.

A lower bound on $\lambda$ already exists from standard Big Bang Nucleosynthesis $(\mathrm{BBN})$ which implies $\Omega_{\phi}(\mathrm{MeV})<0.045$, or, considering a possible underestimation of systematic errors, the more conservative result $\Omega_{\phi}(\mathrm{MeV})<0.09$ 17; these bounds require, respectively, $\lambda>9$ and $\lambda>6.5$ for the model we are considering.

We conclude that, with $n_{s} \approx 1$, the $\Lambda$ CDM model becomes increasingly disfavoured compared with this quintessence model as the amount of early quintessence becomes higher $(\lambda \lesssim 15)$. For $n_{s}<1$, the opposite is true i.e $\Lambda$ CDM is favoured as compared to quintessence if $\lambda \lesssim 15$. Notice that, as $\lambda$ increases $(\lambda \gtrsim 18)$, independently of the value of $n_{s}$, the model's results become comparable to $\Lambda$ CDM's, as should be expected since $\bar{\Omega}_{\phi}^{l s}$ decreases. Moreover, quintessence is distinguishable from $\Lambda$ CDM only for $h<0.73$ and $n_{s} \approx 1$.

Finally, we would like to mention that the non-negligible fraction of dark energy at last scattering and during structure formation we obtain for this model, typical of early quintessence models, will lead to suppressed clustering power on small length scales as suggested by WMAP/CMB/large scale structure combined data 16 .

\section{Acknowledgments}

NMCS acknowledges Fundação para a Ciência e a Tecnologia (FCT) under grant SFRH/BD/4797/2001 and Grupo Teórico de Altas Energias (GTAE) for travel

rioproc2: submitted to World Scientific on December 16, 2018 
support.

\section{References}

1. B. Ratra, P.J.E. Peebles, Phys. Rev. D37 3406 (1988); Ap. J. Lett. 325117 (1988); C. Wetterich, Nucl. Phys. B302 668 (1988); R.R. Caldwell, R. Dave, P.J. Steinhardt, Phys. Rev. Lett. 80, 1582 (1998); P.G. Ferreira, M. Joyce, Phys. Rev. D58,023503 (1998). I. Zlatev, L. Wang, P.J. Steinhardt, Phys. Rev. Lett. 82, 986 (1999); P. Binétruy, Phys. Rev. D60, 063502 (1999); J.E. Kim, JHEP 9905, 022 (1999); T. Barreiro, E. J. Copeland, N. J. Nunes, Phys. Rev. D 61, 127301 (2000); M.C. Bento and O. Bertolami, Gen. Relativity and Gravitation 31, 1461 (1999); M.C. Bento, O. Bertolami, P.T. Silva, Phys. Lett. B498, 62 (2001); O. Bertolami, P.J. Martins, Phys. Rev. D61, 064007 (2000); A.A. Sen, S. Sen, S. Sethi, Phys. Rev. D63, 107501 (2001); A.A. Sen, S. Sen, Mod. Phys. Lett. A16, 1303 (2001).

2. Y. Fujii, Phys. Rev. D61, 023504 (2000) ; A. Masiero, M. Pietroni and F. Rosati, Phys. Rev. D61, 023504 (2000) ; M.C. Bento, O. Bertolami, N. C. Santos, Phys. Rev. D65, 067301 (2002) .

3. A. Kamenshchik, U. Moschella, V. Pasquier, Phys. Lett. 511, 265 (2001); M.C. Bento, O. Bertolami and A.A. Sen, Phys. Rev. D66, 043507 (2002); N. Bilić, G.B. Tupper, R.D. Viollier, Phys. Lett. B535, 17 (2002).

4. K. Freese, M. Lewis, Phys. Lett. B540,1 (2002).

5. M. Doran, M. Lilley, J. Schwindt, C. Wetterich, Astroph. J. 559, 501 (2001).

6. P. de Bernardis et al., Nature 404, 955 (2000); C.B. Netterfield et al., Astrophys. J. 571, 604 (2002); J. E. Ruhl et al., Astrophys. J. 599, 786 (2003).

7. S. Hanany et al., Astrophys. J. 545, L5 (2000); A. Balbi et al., Astrophys. J. 545, L1 (2000) [Erratum-ibid. 558, L145 (2001)].

8. A. Benoit et al. [the Archeops Collaboration], Astron. Astrophys. 399, L25 (2003).

9. D. N. Spergel et al., Astrophys. J. Suppl. 148, 175 (2003).

10. A. Albrecht, C. Skordis, Phys. Rev. Lett. 84, 2076 (2000); A. Albrecht, C. Skordis, Phys. Rev. D66, 043523 (2002).

11. N.A. Bahcall et al., Astrophys. J.585, 182 (2003); T.H. Reiprich and H. Böhringer, Astrophys. J. 567, 716 (2002); S.W. Allen, R.W. Schmidt, A.C. Fabian and H. Ebeling, Mon. Not. Roy. Astron. Soc. 334, L11 (2002); U. Seljak, 2002, MNRAS 337, 769 (2002); P.T.P. Viana, R.C. Nichol and A.R. Liddle, Astrophys. J. 569, L75 (2002).

12. J. Barrow, R. Bean, J. Magueijo, Mon. Not. Roy. Astron. Soc. 316, L41 (2000).

13. S. Hellerman, N. Kaloper, L. Susskind, JHEP 0106, 003 (2001); W. Fischler, A. Kashani-Poor, R. McNess, S. Paban, JHEP 0107, 003 (2001); E. Witten, hep-th/0106109

14. T. Barreiro, M. C. Bento, N. M. Santos and A. A. Sen, Phys. Rev. D 68, 043515 (2003).

15. M. Doran, M. Lilley, Mon. Not. Roy. Astron. Soc. 330, 965 (2002).

16. R. R. Caldwell, M. Doran, C. M. Mueller, G. Schaefer and C. Wetterich, As-

rioproc2: submitted to World Scientific on December 16, 2018 
trophys. J. 591, L75 (2003).

17. R. Bean, S. H. Hansen, A. Melchiorri, Phys. Rev. D64, 103508 (2001).

rioproc2: submitted to World Scientific on December 16, 2018 